\title{
Atomic Resolution Defect Analysis Using Low Angle ADF-STEM
}

\author{
P.J. Phillips ${ }^{1}$, L. Kovarik ${ }^{2}$, and M.J. Mills ${ }^{1}$ \\ Department of Materials Science and Engineering, Ohio State University, Columbus OH 43210 \\ 2 \\ Pacific Northwest National Lab, Richland, WA 99352
}

High-resolution scanning transmission electron microscopy (STEM) has recently been one of the most important techniques in advancing the analysis of crystalline materials. Traditionally, high-resolution STEM is performed in the Z-contrast mode, wherein the annular dark-field (ADF) detector is situated such that only high-angle scattering is accepted. It has been shown that under these conditions, the resulting intensity can be approximated using an incoherent imaging model [1]. Because the low-angle Bragg scattering is not collected by the ADF, the resulting contrast is generally directly interpretable, and does not suffer some of the drawbacks of phase-contrast high-resolution TEM, such as strong dependencies on defocus and thickness [2].

Much of the initial work on Z-contrast STEM was rather ambiguous in defining the "high angle" necessary for incoherent imaging to apply, yet other calculations yielded an inner detector angle so large that the resulting signal would be very weak [1-3]. However, when using a more modest scattering angle as in low angle annular dark-field (LAADF), while still in a zone-axis configuration, enhanced contrast from crystalline defects results. Early work on the origin of this contrast has suggested that atoms, displaced near defects and potentially offset from perfect lattice channels, cause the electron beam to be de-channeled while penetrating the specimen [4-6].

The present contribution highlights LAADF imaging of defects, while maintaining atomic resolution. Specifically, various defects present in a Ni-based superalloy were examined using an FEI Titan $80-300$ probe-corrected monochromated S/TEM operated at $300 \mathrm{kV}$. The camera length (CL), i.e., ADF detector acceptance angle, was systematically varied to show either Z-contrast or defect contrast.

Fig. 1 illustrates the defect contrast obtained in zone-axis oriented LAADF. The left image was taken from the same area as the right, only with the ADF acceptance angle adjusted to $14-91 \mathrm{mrad}(\mathrm{CL}=285 \mathrm{~mm})$; paired dislocations exhibit bright contrast throughout the left image, highlighted with red lines. The right image was acquired with the ADF acceptance angle ranging from $69-422 \mathrm{mrad}(\mathrm{CL}=58 \mathrm{~mm})$, and is considered to be Z-contrast in nature. As shown in Fig. 2, atomic resolution LAADF images remain interpretable, despite the contribution of low-angle scattering to the image. The stacking fault present runs parallel to one set of $\{111\}$ planes, with interplanar spacing $0.206 \mathrm{~nm}$. When the CL is adjusted for Z-contrast (right image), note the lack of enhanced intensity over the fault, indicating that the bright contrast observed in LAADF is not a result of heavy-element segregation to the fault or any variance in chemical species. Since such heavy-element segregation can occur at planar defects, it is particularly important to accurately understand the LAADF contrast, in order to distinguish it from Z-contrast.

Additionally, high-resolution images were acquired in defect-free regions of the crystal. Within the given range of ADF detector acceptance angles, variations on defocus again produced atomically resolved images, free of contrast reversals. Experimental evidence has also indicated that a given defect or strain field need not be perfectly aligned along a low-index zone for observation, a requirement that can complicate traditional HAADF imaging. STEM simulations of both perfect and faulted crystals are in progress and will be discussed in addition to experimental results. 


\section{References}

[1] R.F. Loane et al., Ultramicroscopy 40 (1992) 121.

[2] S.J. Pennycook and D.E. Jesson, Ultramicroscopy 37 (1991) 14.

[3] M.M.J. Treacy, J. Microsc. Spectrosc. Electron. 7 (1982) 511.

[4] J.M. Cowley and Y. Huang, Ultramicroscopy 40 (1992) 171.

[5] J. Liu and J.M. Cowley, Proc. Ann. EMSA Meeting 50 (1992) 1224.

[6] J.L. Lee and J. Silcox, Ultramicroscopy 84 (2000) 65.

[7] Support was provided by the University Strategic Alliance (USA) program, GE Aviation.
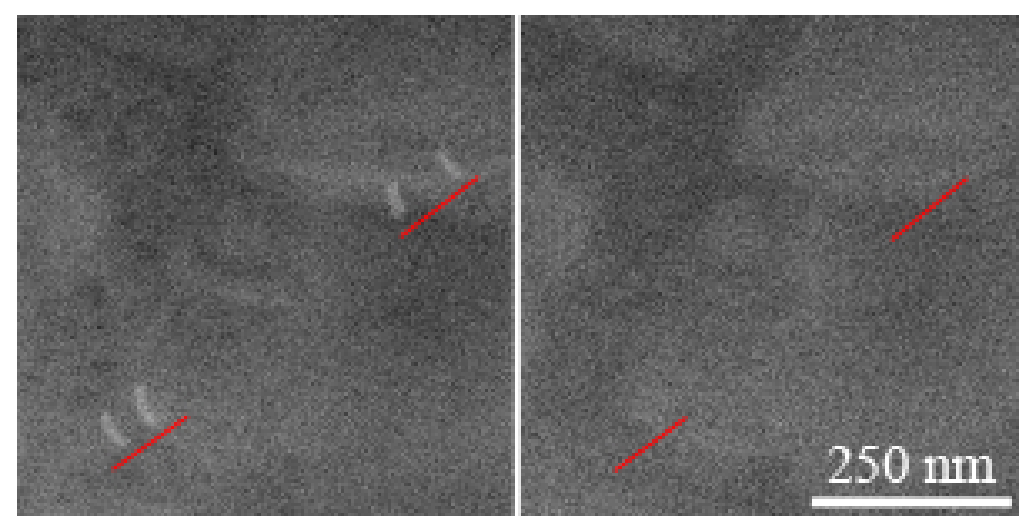

Figure 1: Two images of the same area with the foil tilted to the [011] zone; the left image was acquired at a CL of $285 \mathrm{~mm}$ (LAADF) and defect contrast is visible; when the CL is decreased to $58 \mathrm{~mm}$ (HAADF), Z-contrast results, as in the right image.

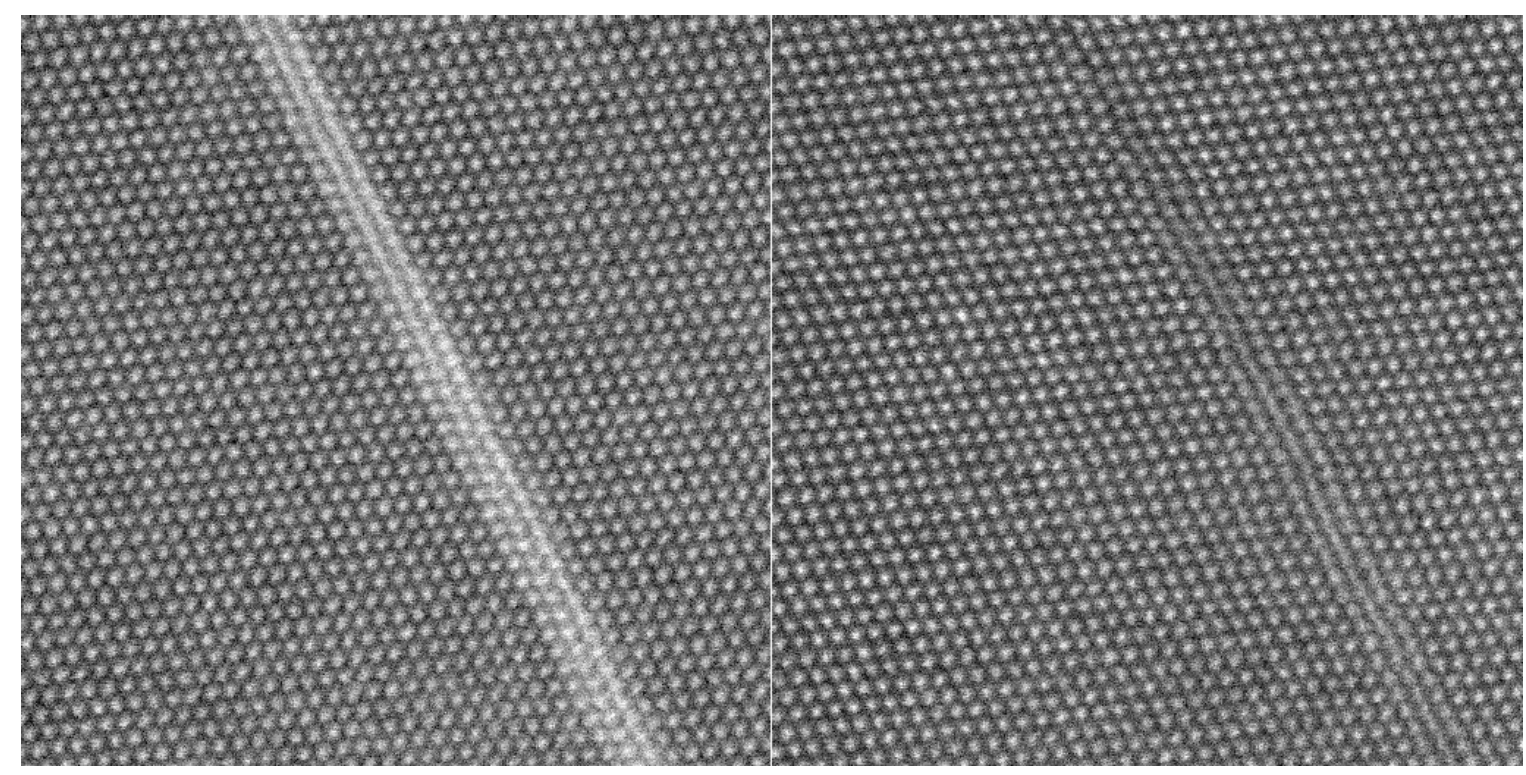

Figure 2: Enhanced contrast from a stacking fault visible in [011] LAADF conditions, CL = $285 \mathrm{~mm}$ (left); the right image, acquired at $\mathrm{CL}=58 \mathrm{~mm}$, eliminates the possibility that the enhanced contrast observed is due to heavy-element segregation to the defect. 